\title{
Assembling Music
}

\author{
Yoichi Nagashima \\ Department of Design, Shizuoka University of Art and Culture, Japan \\ nagasm@suac.ac.jp
}

\begin{abstract}
In this paper, I report on my three projects - sound installation, new musical instruments and new platform of computer music - the common key concept is "assembling music". Musical performance is a kind of expansion of human-body activity. I have been developing many kinds of musical instruments as a part of my composition, and I focused essentially on human "assembling" action in music performance in these projects. Of course, musical composition is an assembling process of musical parts. However, I want to expand the concept of "assembly" - not only for composition but also for performance. I hope this report expands the possibilities in interaction design for media art, and hope to discuss the technological detail and artistic approach.
\end{abstract}

Keywords: musical building block, expanded LittleBitsSynth, assembling performance

\section{Introduction}

Musical performance is a kind of expansion of human-body activity, for example - beating, rubbing, bending, pushing, pulling, repelling, etc. I have developed many kinds of musical instruments as a part of my composition, sometimes with "contactless" interface like a Theremin. But now, as CV (computer vision) using a Webcam or Kinect for music interface is very popular, I do not follow the trend.

In this paper, I will show some experimental works of "Assembling Music". Of course, the musical composition is essentially an assembling process of musical parts. I want to expand the concept of "assembly" - not only for the composition but also for the performance. The human action of assembling something is familiar in many industries, but is beautiful in productions by experts. Children enjoy assembling and disassembling building block toys, because this simple behavior is essential for human culture. In this paper, I report on three experimental works of musical interface - an installation work, new instruments and a new platform for improvisational composition/performance.

\section{Installation work 'Color Orchestra'}

This sound installation (or musical instrument) was created by Ryu Junhee who was an undergraduate student in my seminar. He targeted the "musical building blocks" as a toy, and the system had a total 44 colored wooden blocks and a wooden base machine (playing table).

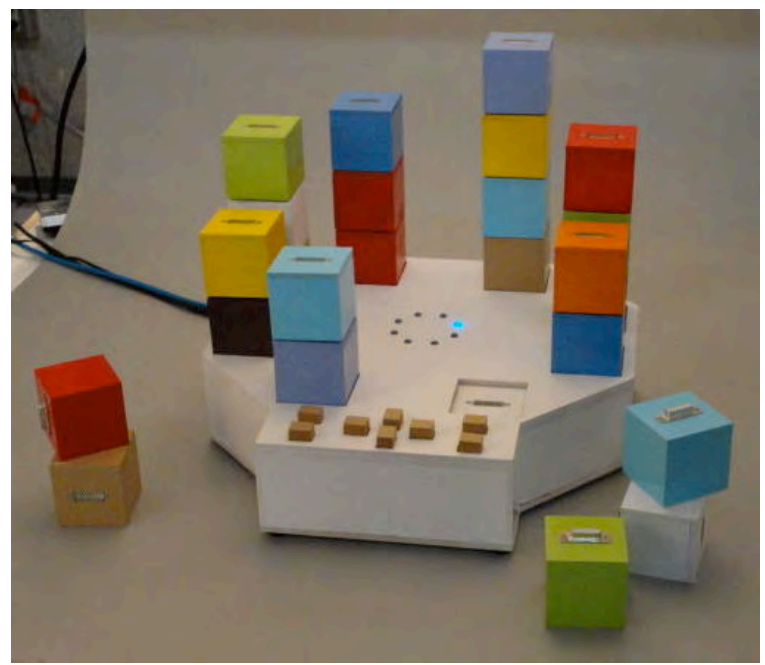

Figure 1. The installation "Color Orchestra" 
Figure 1 shows the system which had a circle of eight bright blue LEDs, flashing in sequence in the center of the table. On the table, there was a shallow recessed square depression outside each of the eight LEDs. The shallow recessed square depression acts as a "connector" for blocks which people can pile up on the table freely.

At first, there are no blocks on the stage, and the blue LEDs flash sequentially as musical tempo alone. If people stack one block on one location, the assigned sound related to the color is generated at the moment the assigned blue LED flashes. Because there are eight points of sound generation, this instrument is "8-beat" sequencer.

People can stack any number of blocks on any location, thus the generated sound is the combination of stacked blocks - four blocks for each of eleven colors which means four percussion (BD, SD, $\mathrm{HH}$, clap) and a seven-note scale (C, D, E, F, G, A, B). If same-color blocks are stacked in the same location, the volume of the assigned sound doubles. People can push other control buttons on the stage of the system - to change the tempo, to change the timbre of scale notes, to change total loudness and to change the depth of echo effect.

Technically, this system is realized by Max/MSP patch connected by microprocessor AKI-H8 inside of the system via MIDI. Each block has a 15-pin DSUB male connector at the bottom of the block and has a 15-pin DSUB female connector at the top of the block. These blocks can be piled up (connected) higher on top of each other and can easily be disconnected. Each block receives the common "address" signal from the system via DSUB connector, and the decoder IC (inside the block) generates an output signal "I am here!" to the common signal line with an open-drain buffer (wired-OR connection).

The superior specification of this system is realtime assembling / disassembling performance of musical blocks - improvisation on the stage. After finishing the creation of this installation work, I composed a new work of computer music using this system as a musical instrument. Figure $\mathbf{2}$ shows the performance of the new work called "Joyful Boxes" on stage.
Behind the stage, the realtime $3 \mathrm{D}$ graphics (created by Open-GL of Max/jitter) was projected on the screen - which is synchronized with the beat of eight blue LEDs of the system.

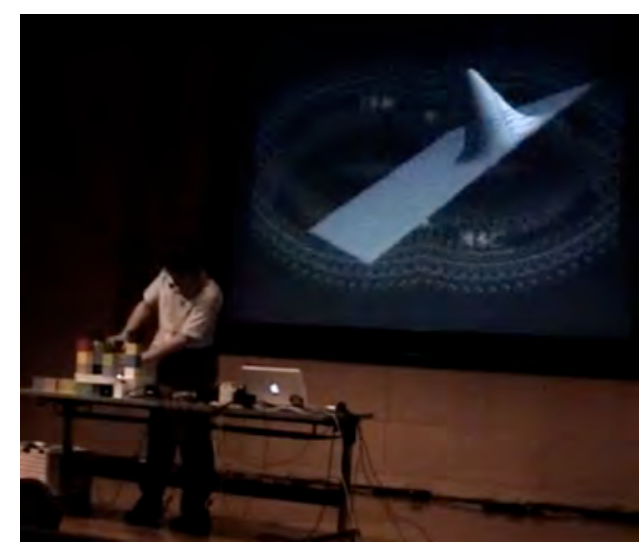

Figure 2. The performance of "Joyful Boxes"

At the beginning part of "Joyful Boxes", I performed like a child playing a block-building toy with the beat of blue LED, making sounds, and moving $C G$ on the screen. As the music scene proceeds, I hold one block and sing/shout something with the microphone then the system works as a sampler of the voice, and the assigned sound of the block changes into my realtime sampled voice. When I pile up the "sampled voice" color blocks, my voice is mixed with other sounds of blocks. This musical performance means "assembling sound itself" and "assembling musical elements" of musical beats with improvisation. You can view this performance on YouTube (Nagashima 2012).

\section{New Instruments "GHI2014"}

This section is a report of a new instrument from GHI project, called "GHI2014". In 2007, I proposed a new concept/project called "GHI" meaning "It might be good that musical instrument shines, wouldn't it?" in Japanese.

I selected three policies while looking straight at the essence of musical instruments as a new approach. (1) Musical instruments are a tool of music, and are good partners for musical expression in the performance. (2) For natural musical instruments, the sound is radiated, and not only the audience but also the 
player receives the sound from the whole space. (3) The player's expression mesmerizes an audience from not only the aural route but also the visual route. The idea of a new project arose here. I produced a new instrument called "Cyber Kendang", and new piece (using it) was selected and performed by the author at NIME07 concert in NYU (Figure 3: Nagashima 2007).

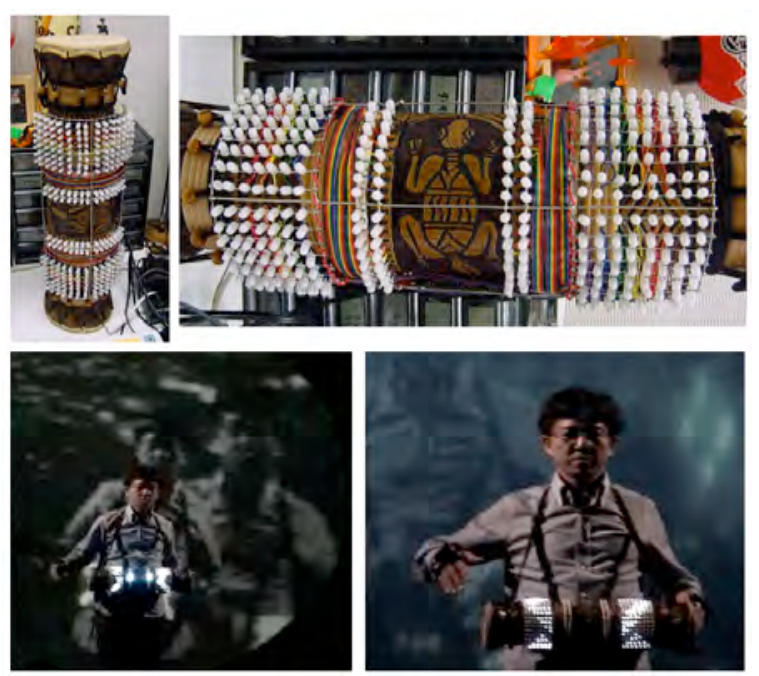

Figure 3. The performance of "Cyber Kendang"

I had reported that the "Propeller" processor is a good platform to realize multimedia interactive systems (Nagashima 2009). In 2013, I supported my student's project - "Shining one-piece dress". Her concept was very simple and cute - to produce a black semi seethrough one-piece dress which shines with many internal white LEDs. I developed a system with Propeller processor as 24-channel LED driver for individual PWM control (Figure 4). I used the reel-type LED ribbon, which contains 3 powerful white LEDs per $5 \mathrm{~cm} /$ block. The 24 output ports were driven by $2 \mathrm{SC}_{18} 15$ for $12 \mathrm{~V} 6 \mathrm{omA}$ (3 LED) or $12 \mathrm{~V}_{12} \mathrm{omA}$ (6 LED) each, and controlled by fast PWM driver module (originally developed).

Inspired by that project, bright LED ribbons and Propeller PWM driver, I have decided to start "GHI" again. I aimed for a new instrument with the same concept, self-many-shining instrument called "GHI2014". The dress had a total of 304 LEDs, but "GHI2014" has a total of 864 strong LEDs. The impact of this light is very expressive, and sometimes performers use sunglass on stage. In addition to this, I aimed to add one idea. I have developed many other instruments and brought them on world tours, and my big theme was the portability of instruments. Small instruments/interfaces like iPhone are easy to carry, but bigger-scale instruments have more effect/impact on stage, so this is the essential opposition.

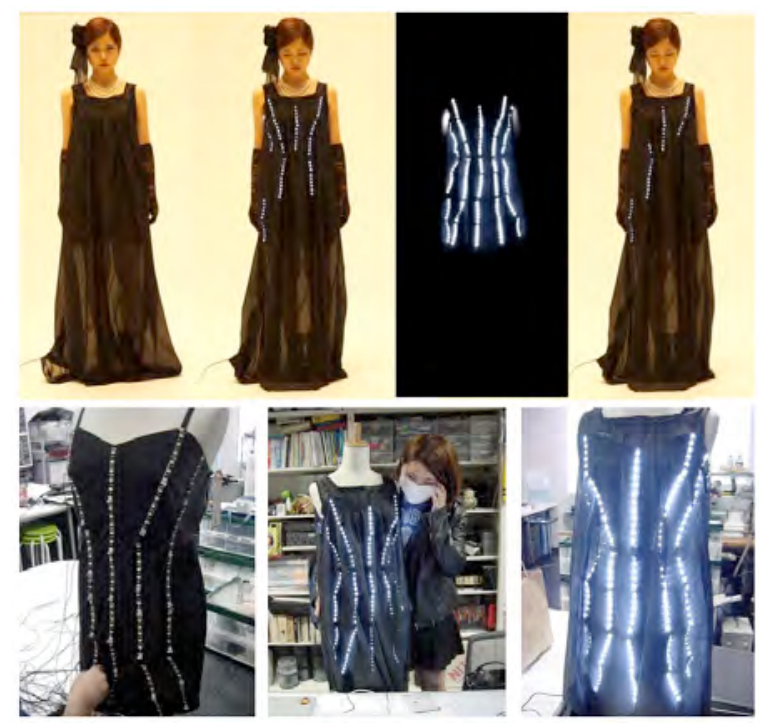

Figure 4. The "Shining one-piece dress"

A good idea came to me - an instrument which can be carried while decomposed and can be assembled/used. This disassembly/assembly process was a good concept for my composition using it later. Eventually the overall shape of this instrument became two 12 ridges outlining the shape of an octahedron side-by-side. Each ridge line is a square pillar which has 15 LEDs on each of 4 surfaces. All edges of these ridge lines are separable, with small strong Neodymium magnets. The LED blocks of the total 24 ridge lines of the double octahedron are realtime controlled with PWM via MIDI. Around this sculpture, 6 ultrasonic range finder sensors can be setup, and the output is also MIDI. Figure $\mathbf{5}$ shows the shape of "GHI2014".

Eventually, the GHI2014 contained two Propeller drivers. The interesting specification of the GHI2014 are the three modes of the interface - standalone mode, sensor mode and instrument mode. In the standalone mode, the 1st Propeller receives the distributed output from the 2nd Propeller (sensors), and reacts 


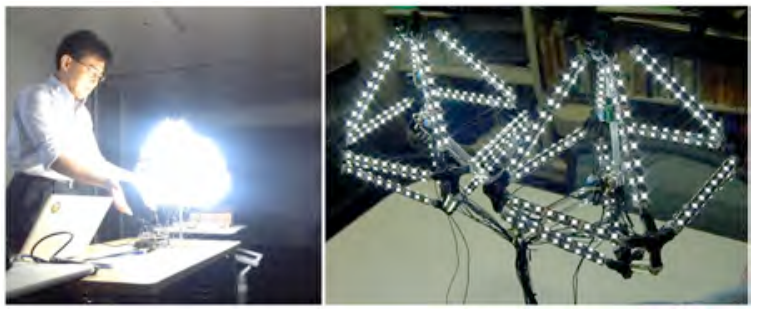

Figure 5. New Instruments "GHI2014"

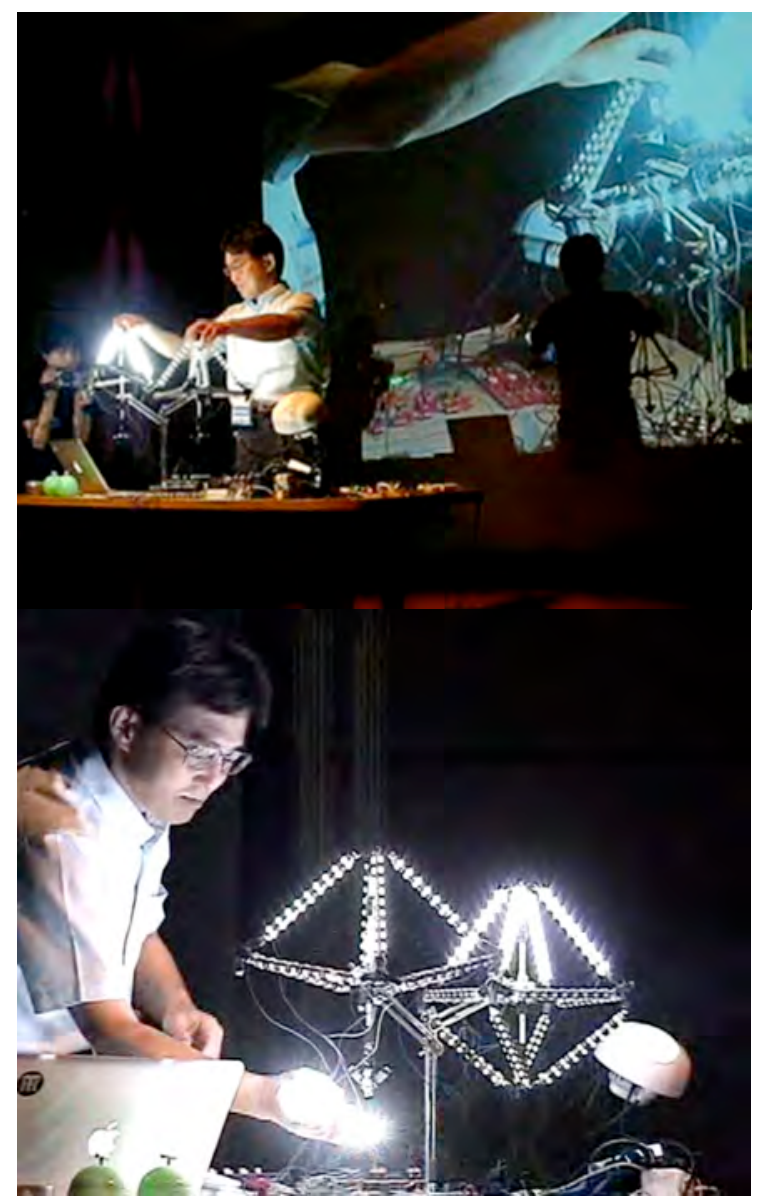

Figure 6. The performance of "GHI2014_Ogaki"

without MIDI input. So, GHI2014 works without a computer, works as an installation - detects human action and shines by itself. In the sensor mode, with all LED cables disconnected, GHI2014 works as six-channel ultrasonic range finder sensors (removable/re-settable) for other applications as interactive installations. In the instrument mode, the 1st Propeller can cut the distributed output from the 2 nd Propeller by special MIDI command, GHI2014 works with the concept of "GHI", and shining pattern can be programmed with realtime generated sound/music/graphics performances.
I have composed a new work featuring this new instrument in 2014 (Nagashima 2014). At the beginning of the work "GHI2014_Ogaki", I performed with the "expanded LittleBitsSynth" (explained later) and started assembling this instrument. With the realtimegenerated BGM (Rhythm section), each part of the instrument was flashing synchronized with the beat, and I connected them to construct the shape on stage. After the whole shape was built, I attached the six ultrasonic distance sensors, then the total 24 ridge lines of the double octahedron started shining with my performance, as controlling the Theremin. This assembling performance was an important part of the work, so the live camera image (captured by staff) was projected on the screen behind the stage. From this projection, the audience were able to understand the relation between the assembling and the sound. Figure 6 shows the performance on stage.

\section{The "expanded LittleBitsSynth"}

In 2013, the release of "LittleBitsSynth" (http://littlebits.cc/kits/synth-kit) was an exciting development in both the Computer Music community and the Sketching (Physical Computing) community. I arranged the "LittleBitsSynth" for expanded expression in computer music as a concept of "Assembling Music", and I will introduce the details here. Figure 7 shows the performance.

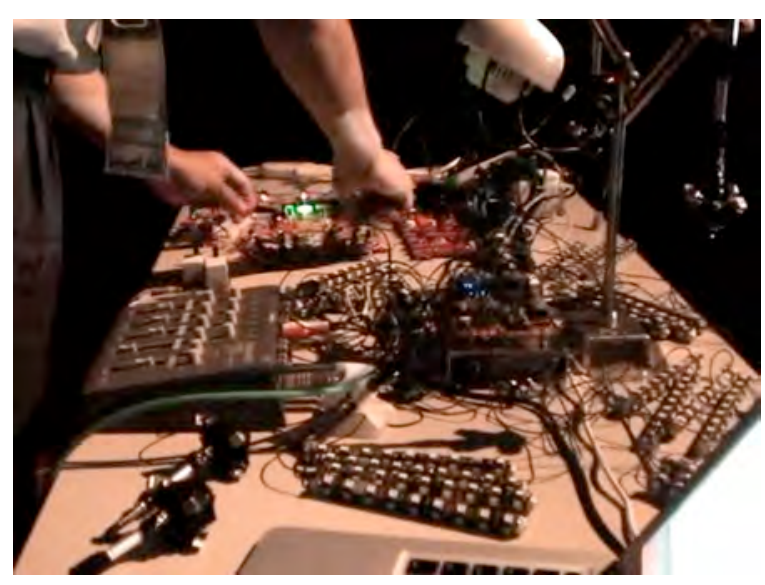

Figure 7. Performance with "LittleBitsSynth" 
At first, I cut the connecting cables and inserted male-female a "pin header" connector between them. Then, I connected cables from three types of sensors (infra distance sensor, 2D acceleration sensors, 3D acceleration sensors) to the "pin header" connectors. Thus, I could insert special external sensors, or could pass through (directly connecting). Figure $\mathbf{8}$ shows the close-up.

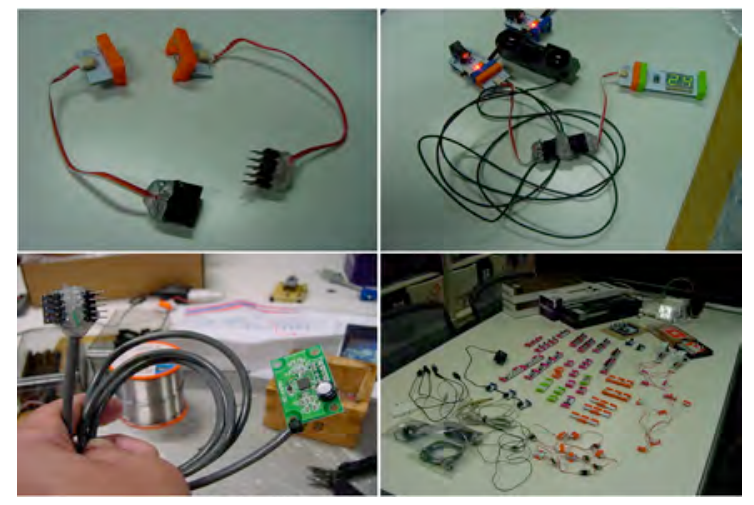

Figure 8. Inserting sensors/connectors

Next, I arranged the "LittleBits Arduino" (Figure 9) for usefulness/controllablility in the system. The "LittleBits Arduino" has [3 input and 3 output] voltage ports using the "bitSnap" magnetic connector, and pin holes of other ports on the board - compatible with standard Arduino. We can program it with the Arduino IDE - like Arduino UNO, Arduino Pro, etc. However, the programmed Arduino works only as a standalone system, not as a peripheral system with Max environment. Therefore, I replaced the firmware of "LittleBits Arduino" with Firmata (http://firmata.org) which is a protocol for communicating with microcontrollers from software on a computer. The protocol can be implemented in firmware on any microcontroller architecture as well as software on any computer software package.

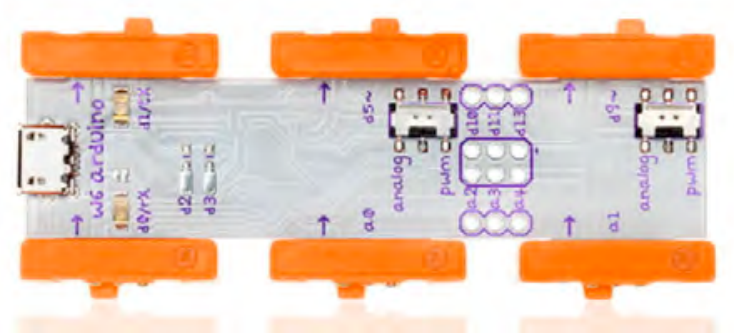

Figure 9. LittleBits Arduino
Then, I arranged the Max object/patch "maxuino" (http://maxuino.org) as a simple/useful tool. Figure 10. Upper) shows the default maxuino patch, but I analyzed and modified the internal "maxuino.js" object, and finally produced the original "3 Arduino controller" maxuino-like patch (Figure 10. Lower). In this way, we can control the "LittleBitsArduino running Firmata" by using "arranged maxuino" just like as Gainer.

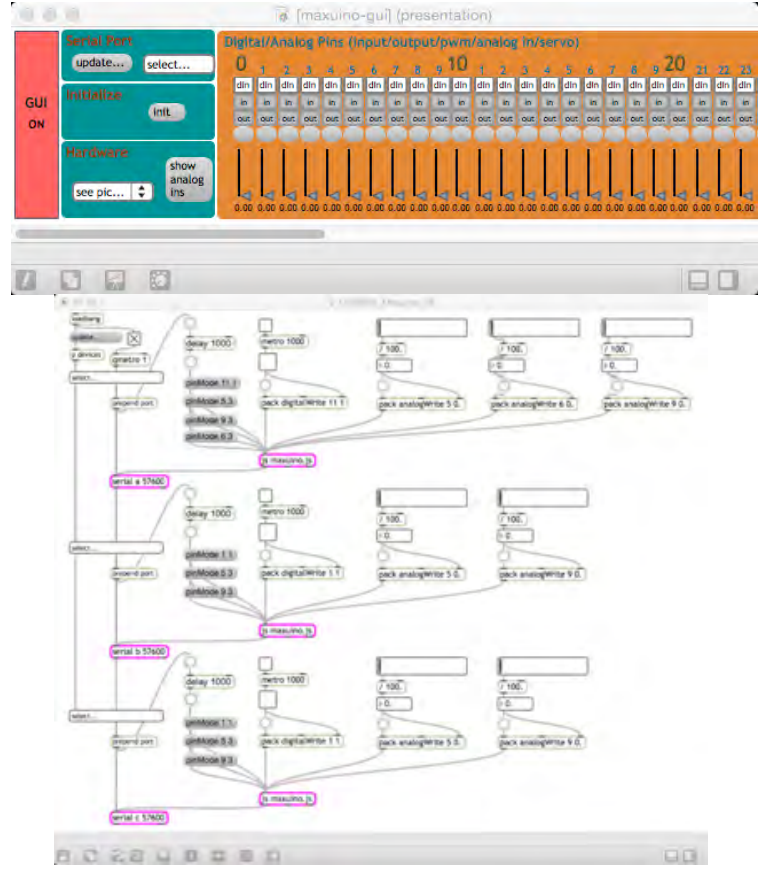

Figure 10. The "maxuino" (default vs. arranged)

The "LittleBits-Arduino(Firmata)" and "arranged maxuino" was a very smart combination with Max, but there was a serious problem - the magnetic connector of LittleBits. The "bitSnap" magnetic connector is a very smart and funny idea for our "Joy of Assembly/Disassembly", and the "slight" momentary contact failure is almost OK for voltage connection as sensor (control), sound (pitch, amplitude, waveform). Humans cannot recognize the momentary skip of the voltage. On the other hand, the "LittleBits-Arduino (Firmata)" and the "arranged maxuino" system are undermined by a serious obstacle, because the momentary contact failure causes the RESET of Arduino, and the Max patch (maxuino) loses sight of the "serial" object and requires a restart of the patch. Of course, in the music scene, this is not acceptable. 
Based on my experience and knowledge of computer music over 20 years, the most reliable connection is MIDI. Thus I decided to develop a new system to control LittleBitsSynth system from Max without "LittleBits-Arduino (Firmata) + arranged maxuino". This system was constructed with new a microcontroller "mbed" - NucleoF401RE - ARM processor developed by open source tool on the Web. Figure 11 shows the system - the upper left is six channel voltage output ports, upper right is 10 channel voltage input ports, lower left is MIDI IN/OUT and three channel trigger output ports and lower right is my wiring. With this system, the realtime control is widely expanded with LittleBitsSynth and the reliability is very good. This system is robust and tough for improvisational assembling and disassembling performance on stage. I have plans for composing and performing in the future using this system.

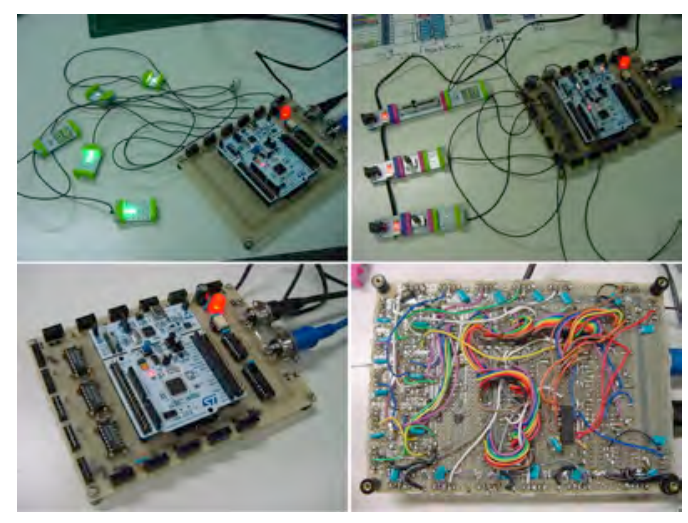

Figure 11. LittleBits Controller (mbed)

\section{Demonstration}

After this paper was accepted by the organizer of Soundislands Festival, I was requested to give an additional demonstration presentating my instruments at the ArtScience Museum on Sunday. I am planning three demonstrations there.

The first demonstration will be a live performance of "GHI2014". I will construct a musical instrument during the music performance time, and perform live sound/light generation.
The second demonstration will be a live lecture of "LittleBits-Arduino(Firmata)" and "LittleBitsSynth controller by mbed". I will show some techniques of the Open Source Culture in computer music.

The third demonstration - somewhat related to my theme - will be the recent two novel instruments. One is the "Double Myo" sensors, and the other is a "New tactile instrument which has not yet been named". Both will be accompanied by a simple performance. In particular, the latter instrument has 10 unique tactile sensors, and it is a useful case study of "mbed" as a controller.

\section{Conclusion}

I have reported on my three projects - sound installation, new musical instruments and a new platform for computer music. The common key concept is "assembling music". I hope to expand the concept of "assembly" - not only for composition but also for performance. I hope this report expands the possibilities in interaction design for media art.

\section{References} es".

Yoichi, Nagashima. (2012). Piece "Joyful Boxhttp://www.youtube.com/watch?v=GiOohVnoAoc

Yoichi, Nagashima. (2007). "GHI project and [Cyber Kendang]". Proc. NIME 2007.

Yoichi, Nagashima. (2007). "GHI Project : New Approach for Musical Instrument". Proc. ICMC 2007.

Yoichi, Nagashima. (2009). "Parallel Processing System Design with [Propeller] Processor". Proc. NIME 2009.

Yoichi, Nagashima. (2014). Piece "GHI2014". URL

http://www.youtube.com/watch?v=sRhxdGaSOfE, http://www.youtube.com/watch?v=c1e1U4tTkcU, http://www.youtube.com/watch?v=wh_iztSy-B8 